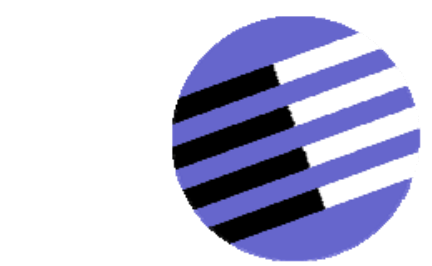

GOVERNANCE AND THE EFFICIENCY

OF ECONOMIC SYSTEMS

GESY

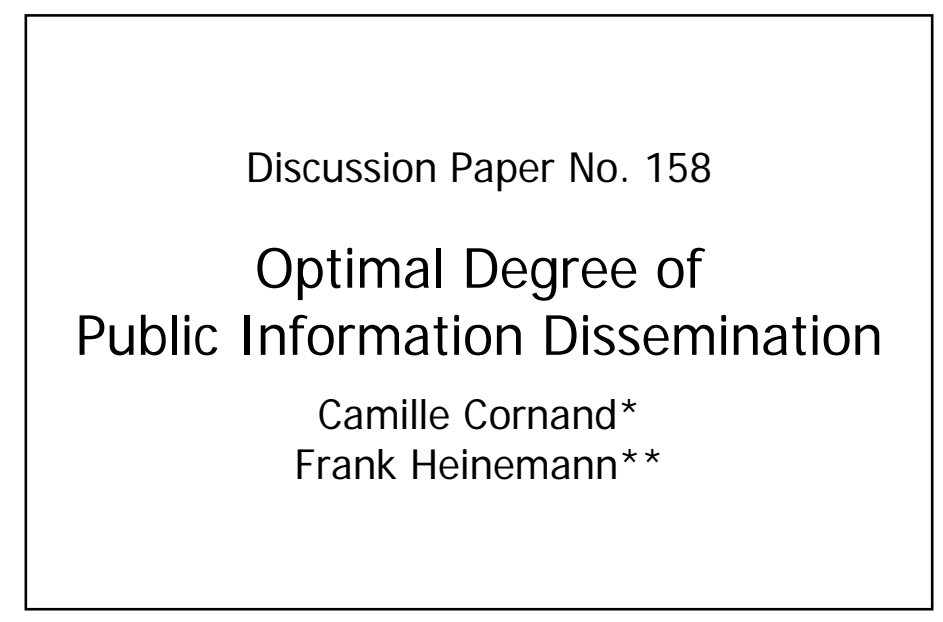

February 2006

*Camille Cornand, Financial Market Group, London School of Economics and Political Sciences

Houghton Street, London WC2A 2AE, United-Kingdom, C.Cornand@lse.ac.uk

**Frank Heinemann, Technische Universität Berlin, Sekretariat H 52 Strasse des 17. Juni 135, 10623 Berlin

f.heinemann@ww.tu-berlin.de

Financial support from the Deutsche Forschungsgemeinschaft through SFB/TR 15 is gratefully acknowledged.

Sonderforschungsbereich/Transregio $15 \cdot$ www.gesy.uni-mannheim.de 


\title{
Optimal Degree of Public Information Dissemination
}

\author{
Camille Cornand and Frank Heinemann*
}

February 21, 2006.

\begin{abstract}
Financial markets and macroeconomic environments are often characterized by positive externalities. In these environments, transparency may reduce expected welfare from an ex-ante point of view: public announcements serve as a focal point for higher-order beliefs and affect agents' behaviour more than justified by their informational contents. Some scholars conclude that it might be better to reduce the precision of public signals or entirely withhold information. This paper shows that public information should always be provided with maximum precision, but under certain conditions not to all agents. Restricting the degree of publicity is a better-suited instrument for preventing the negative welfare effects of public announcements than restrictions on their precision are.
\end{abstract}

\section{Keywords}

Transparency, public information, private information, coordination, strategic complementarity.

\section{JEL Classification}

C73, D82, F31.

\footnotetext{
* Camille Cornand, Financial Market Group, London School of Economics and Political Sciences, Houghton Street, London WC2A 2AE, United-Kingdom, C.Cornand@lse.ac.uk and Frank Heinemann, Technische Universität Berlin, Strasse des 17. Juni 135, 10623 Berlin, Germany, f.heinemann@ww.tu-berlin.de. We would like to thank Jean-Pierre Allegret, Romain Baeriswyl, Agnès Bénassy-Quéré, Philippe Martin, Hyun Shin, Anne Sibert, as well as two anonymous referees and the editor of The Economic Journal, Leonardo Felli, for helpful comments. The first author acknowledges support from the U.K. ESRC.
} 


\section{1 - Introduction}

There is a general presumption that higher central bank transparency in terms of information disclosure to a wider audience improves the effectiveness of monetary policy and is beneficial to markets as it reduces information asymmetries, helps to better assess risk and arrive at more informed decisions, thus enhancing market efficiency. While practitioners (in central banks and international institutions) agree on the desirability of informative announcements and promote higher transparency on the ground that any information is valuable to markets, recent academic literature distinguishes private and public information and argues that public announcements may destabilize markets and reduce efficiency by their effects on higher-order beliefs, if markets are characterized by positive externalities. Morris and Shin (2002) (henceforth M-S) have shown that noisy public announcements may be detrimental to welfare from an ex-ante point of view. They conclude that a commitment to withholding relevant information or deliberately reducing its precision may be welfare enhancing.

In this paper we challenge this conclusion by distinguishing two components of transparency: precision of information and degree of publicity. The degree of publicity is the proportion of agents who receive a signal. Using a stylised coordination game introduced by M-S, we show that information should always be provided to the market with maximum precision, but, under certain conditions, the mechanisms of disseminating information should aim at limiting the degree of publicity by providing information only to some fraction of market participants. The optimal degree of publicity depends on the precision of announcements. If there are inevitable limits to the precision, the optimal degree of publicity is rising in precision and reaches one if precision is sufficiently high. The rationale for our results is that partial publicity increases expected utility for those, who receive the information, while potentially detrimental effects on higher-order beliefs are limited if agents expect that others do not possess the same information.

The literature distinguishes at least three different lines of arguments against central bank transparency. Within a Barro-Gordon framework, the inflation bias may be affected either way by policy transparency. In addition, economic transparency may offset desired real effects of monetary policy. Geraats (2002) gives an excellent overview of the pros and cons of transparency with several examples of welfare reducing information in a Barro-Gordon framework. Faust and Svensson (2001) show that complete transparency - that is the observation of the central bank's intentions and goals by the private sector - leads to the largest inflationary bias. Since private agents do not need to infer the preferences of the 
central bank from its behaviour, the monetary authority bears no reputation costs if it pursues hidden objectives in addition to the inflation target. In a model with no inflationary bias and no trade-off between credibility and flexibility, Cukierman (2001) stresses that when the central bank tries to achieve two goals, limited transparency improves the expected social welfare. The central bank can use its private information to attain better values for these goals. Even from a practical point of view, too much transparency of monetary policy can be undesirable. Mishkin (2004) shows that transparency can go too far in complicating the communication process and perturbing central banks' main objectives. Following Winkler (2000), the main drawback of this strand of literature is to underestimate the impact of informational policy on higher-order beliefs.

The discussion about the distinct effects of public and private information started with the application of the theory of global games to speculative attacks by Morris and Shin (1998, 1999). A speculative-attack game has a unique equilibrium if private information is sufficiently precise in relation to public information (Hellwig, 2002a). Heinemann and Illing (2002), Metz (2002) and Bannier and Heinemann (2005) analyse how the ex-ante probability of currency crises is affected by the relative precision of public and private information. To the extent that financial markets exhibit strategic complementarities, common knowledge amplifies the impact of new information and provokes runs into or out of a market, because of higher-order beliefs. Precise public announcements may induce self-fulfilling beliefs and trigger a crisis. Heinemann and Illing (2002) conclude that the central bank should provide information to agents in private with small idiosyncratic noise.

M-S present a stylised coordination game with a unique equilibrium in which public information may be detrimental to welfare if its precision is limited by inevitable forecast errors. Their model emphasizes the role of public information as a focal point for private actions. Strategic complementarities provide incentives to coordinate on the publicly announced state of the world and neglect private information. If public announcements are inaccurate, private actions are drawn away from the fundamental value. Public information is a double-edged instrument: it conveys valuable information, but the desire to coordinate leads agents to condition their actions stronger on public announcements than is optimal. Both effects get stronger if the precision of public information rises. The welfare effect of precision is U-shaped and an infinite precision of public information maximizes welfare. However, if its precision has an upper limit, it may be better not to provide any public information at all than disseminating information with maximum precision. M-S conclude that a welfare maximizing 
information provider might want to reduce the precision of public signals or avoid them entirely.

Amato, Morris and Shin (2002) interpret the model by M-S as a Lucas' islands economy. Building up on this interpretation, Amato and Shin (2006) argue that over-reactions of prices to public announcements raise doubts on the relevance of prices as indicators of economic balances. Morris and Shin (2005) argue that when the central bank conveys its own view clearly, the market's expectations will be closer to the central bank's own expectations instead of correcting eventual faults of the central bank, and market prices will be informationally inefficient. Hellwig (2002b) shows that a higher degree of transparency - meaning a higher degree of common knowledge - reduces the efficiency of monetary policy.

Angeletos and Pavan (2004) and Hellwig (2005) challenge the conclusions of M-S. Considering economies with increasing returns to scale (Angeletos and Pavan) or monopolistic competition (Hellwig), they find that the precision of public information is always welfare increasing. The reason for this is a different notion of individual utility. In M$S$, the payoff of a player decreases with the distance between his own action and the action of others, but this distance is irrelevant from a social perspective. As Angeletos and Pavan (2004, p.3) put it: “more transparent public information facilitates more effective coordination, which is valued by the market but not by the society". Instead, they consider environments in which there is complementarity at the social level so that coordination is socially valuable. However, financial markets are better characterized by coordination games, in which it is socially desirable to avoid any form of overreaction, so that it is always better to evaluate an asset in terms of the fundamental state of the economy rather than the beliefs of market participants.

In a comment, Svensson (2005) suggests that M-S make in fact a good case in favour of transparency. The numerical condition under which an increasing transparency reduces welfare requires that the maximum precision of public information is smaller than the precision of private information, which seems unreasonable, given recent evaluations of public versus private-sector forecasts on GNP growth or inflation (Romer and Romer, 2000). ${ }^{1}$ However, Morris, Shin and Tong (2005) show that if private and public signals are correlated, the potentially welfare reducing effects of public information occur even if public information is more precise than private signals.

\footnotetext{
${ }^{1}$ See also Woodford (2005) for a discussion on this issue.
} 
In analysing the effects of public announcements, most of the literature has concentrated on the optimal precision of public information, generally yielding "bang-bang solutions", where public information should either be as precise as possible or be avoided entirely. Revisiting the beauty-contest framework of M-S, this paper explores a new dimension of information dissemination: the degree of publicity, by which we mean the proportion of economic agents who receive a message. While all of the previous papers distinguish two extreme kinds of signals, messages that are received by single agents only (private information) and messages that are common knowledge to all agents (public information), in the present paper, we allow for intermediate degrees of publicity: messages that are common knowledge to only a fraction of all agents. It turns out that such an intermediate degree of publicity is optimal if the maximum precision of information is sufficiently small.

In situations where public information may be detrimental by coordinating agents' activities away from social optimum, a message of high precision but limited publicity is superior to pure public information with low precision: for those who receive the signal, a high precision of information about underlying fundamentals enhances efficiency of private decisions. A low level of publicity, however, reduces incentives to over-react to this signal and limits the welfare reducing effect on higher-order beliefs: it combines the positive effects of valuable information for those who get it with a confinement of its threats by limiting the number of receivers. Hence, we show that it may be optimal to provide information with an interior degree of publicity either by informing only predetermined agents or by informing agents at random with a probability below one. This partially public information should always be provided with maximum feasible precision.

The central bank has two different tools for conducting informational policy: the precision of information and the level of information dissemination (also called degree of publicity). Both instruments are double-edged: higher precision improves the quality of private decisions by those who receive information and higher publicity enlarges the number of those who benefit from information. At the same time, both instruments raise incentives to overweigh public signals. Our main result shows that a restriction to the number of people receiving public signals is a more efficient tool for avoiding precarious coordination effects than the ambiguity of signals is.

Our results imply that it may be more efficient to disseminate information in communities or through media that reach only a part of all traders. Partially public announcements can also occur when central bankers deliver speeches or invite a small group of journalists (Walsh, 
2005). Announcements in such environments are less widely reported than formal announcements or require more time to penetrate the whole community. Slow penetration prevents that these announcements become common knowledge at any point in time although the propagation of information may raise the degree of publicity above the primary proportion of informed traders. Optimal information policy must account for this multiplier effect. Thereby, our results give a rationale for the common practice of central banks to release partially public information in addition to official publications: information with low precision should be partially withheld from the public. Information of high precision should always be released with full publicity.

The rest of the paper is structured as follows. Section 2 presents the model. Section 3 solves for the unique equilibrium and establishes the position of the model by M-S as a particular case of our framework. Section 4 gives the welfare analysis. Section 5 discusses means by which the central bank can achieve partial publicity and whether private agents might counteract the central bank's intentions and further disseminate its announcements. Section 6 concludes.

\section{2 - The Model}

Our framework is based on M-S who describe a reminiscence of Keynes' "beauty contest" example. Whereas they assume that public announcements are received by all agents and the information provider can choose the precision of public signals, we enlarge the choice set of the information provider by adding a second dimension: the degree of publicity that we model by the fraction of agents who receive a signal. We allow for two schemes of providing information: public signals may be released to a predetermined group of agents or to each agent with some probability. The first interpretation accounts for the possibility of central banks to spread news in certain communities or in a language that is understood only by some agents. The second interpretation is more related to the practical difficulties in achieving common knowledge: public announcement may be spread through media, but each market participant acknowledges a certain medium only with some probability. These probabilities may differ for different media, so that a central bank can choose the degree of publicity by 
selecting appropriate media for publication. Within our model, both schemes of information provision are equivalent. $^{2}$

\subsection{A beauty contest framework}

The model is a principal-agent two-stage game in which the central bank (principal) determines the optimal precision and the degree of publicity that maximise welfare before speculators (agents) take their decision. There is a continuum of agents, indexed by the unit interval [0,1]. Agent $i$ chooses an action $a_{i} \in \mathfrak{R}$, and we write $a$ for the action profile over all agents. The payoff function for agent $i$ is given as in M-S by

$$
u_{i}(a, \theta) \equiv-(1-r)\left(a_{i}-\theta\right)^{2}-r\left(L_{i}-\bar{L}\right) \text {, }
$$

where $\theta$ is the fundamental state of the economy and $r$ is a constant, such that $0 \leq r \leq 1$ and

$$
L_{i} \equiv \int_{0}^{1}\left(a_{j}-a_{i}\right)^{2} d j, \quad \bar{L} \equiv \int_{0}^{1} L_{j} d j .
$$

The utility function for individual $i$ has two components. The first component is a standard quadratic loss in the distance between the underlying state $\theta$ and his action $a_{i}$. The second component is the "beauty contest" term. The loss is increasing in the distance between $i$ 's action and the average action of the whole population. The parameter $r$ is the weight attributed to this strategic uncertainty: the higher $r$ is, the higher is the external effect arising from the coordination motive of decision makers.

However, this spillover effect is socially inefficient and disappears at the social level. Therefore, there may be a conflict between individual decisions and the socially optimal solution. Social welfare is defined as the (normalized) average of individual utilities, given by

$$
W(a, \theta) \equiv \frac{1}{1-r} \int_{0}^{1} u_{i}(a, \theta) d i=-\int_{0}^{1}\left(a_{i}-\theta\right)^{2} d i .
$$

As a consequence, the social planner, who cares only about social welfare, seeks to keep all agents' actions close to state $\theta$.

The payoff structure is a reminiscence of Keynes’ beauty contest. Speculators gain from predicting the average opinion better than others. The motive for predicting others' actions

\footnotetext{
${ }^{2}$ Sims (2003) and Reis (2004) consider firms that have limited capacities to process information or face costs of acquiring, absorbing and processing information. Our partial release of information might also be understood as all firms observing the announcement but only a fraction $P$ actually incorporating the new information into their decision.
} 
arises from a potential redistribution between speculators. Therefore, the "beauty contest"part of the payoff function describes a zero-sum game. Coordination affects individual payoffs but not social welfare.

\subsection{Structure of uncertainty and timing of the game}

Agents face uncertainty concerning $\theta$. However, to decide on an action, they potentially receive two kinds of signals that deviate from $\theta$ by independent error terms with normal distributions. Each agent receives a private signal

$$
x_{i}=\theta+\varepsilon_{i} \text { with } \quad \varepsilon_{i} \sim N(0,1 / \beta)
$$

Signals of distinct individuals are independent and the distribution of private signals is treated as exogenously given. Eventually, agents have access to a public signal

$$
y=\theta+\eta \quad \text { with } \quad \eta \sim N(0,1 / \alpha) .
$$

The public signal is given to each agent with some probability $P$. Since we have a continuum of identical agents, the fraction of agents who receive public information equals $P$ almost certainly. Without loss of generality, we may assume that agents $i \in[0, P]$ receive the public signal and agents $i \in(P, 1]$ must rely on their private signals only. The signal $y$ is "public" in the sense that the actual realization of $y$ is common knowledge among agents $i \in[0, P]$. Parameters $\alpha$ and $\beta$ are the precisions of public and private signals.

The optimal action of agent $i$ is given by the first order condition:

$$
a_{i}=(1-r) E_{i}(\theta)+r E_{i}(\bar{a})
$$

where $E_{i}($.$) is the expectation operator of player i$ and $\bar{a}=\int_{0}^{1} a_{j} d j$ is the average action in the population. The following expressions come straightforwardly:

- The expected state for an agent who does not receive $y$ but possesses his own private information is given by $\mathrm{E}\left(\theta \mid x_{i}\right)=x_{i}$ and his expected average action is given by

$$
E\left(\bar{a} \mid x_{i}\right)=x_{i} .
$$

- The expected state for an agent who receives $y$ on top of his own private signal is

$$
E\left(\theta \mid y, x_{i}\right)=\frac{\beta x_{i}+\alpha y}{\alpha+\beta}
$$


and we also have his expectation of the others' signals,

$$
E\left(x_{j} \mid x_{i}, y\right)=E\left(\theta \mid y, x_{i}\right)=\frac{\beta x_{i}+\alpha y}{\alpha+\beta}
$$

The game consists of two stages. First, the principal (central bank) chooses the level of public information disclosure $P$ and its precision $\alpha$ in order to maximize expected welfare. Then, in the second stage, agents choose their actions $a_{i}$ maximizing expected utility. An equilibrium of the game consists of strategies for the central bank and for the continuum of speculators such that no player has an incentive to deviate. First, we solve the subgame of the second stage for a given combination of $P$ and $\alpha$.

\section{3 - Equilibrium}

Agents who do not receive the public signal choose $a_{i}=x_{i}$. For the normal distribution, all conditional expectations are linear combinations of available information. The first order condition shows that the optimal action is a linear function of conditional expectations. Thereby, the optimal strategy of any agent who receives the public signal $y$ is a linear strategy of the form

$$
a_{j}=\gamma x_{j}+(1-\gamma) y
$$

A formal proof is given in Appendix A. The optimal weight $\gamma$ depends on an agent's expectations about the behaviour of other players. Because the best response of any agent is unique, in equilibrium, all players choose the same $\gamma$. The conditional estimate of the average action across all agents is then given by

$$
E(\bar{a})=P\left[\gamma E\left(x_{j}\right)+(1-\gamma) E(y)\right]+(1-P) E\left(x_{j}\right) .
$$

For any agent $i$ who receives both signals:

$$
\begin{aligned}
E\left(\bar{a} \mid x_{i}, y\right) & =P\left[\gamma E\left(x_{j} \mid x_{i}, y\right)+(1-\gamma) y\right]+(1-P) E\left(x_{j} \mid x_{i}, y\right) \\
& =P(1-\gamma) y+(P \gamma+1-P) E\left(x_{j} \mid x_{i}, y\right) \\
& =P(1-\gamma) y+(P \gamma+1-P) \frac{\beta x_{i}+\alpha y}{\alpha+\beta} .
\end{aligned}
$$

Thus, agent $i$ 's optimal action, for $i \in[0, P]$, is given by 


$$
\begin{aligned}
a_{i} & =r E_{i}\left(\bar{a} \mid x_{i}, y\right)+(1-r) E_{i}\left(\theta \mid x_{i}, y\right) \\
& =\frac{x_{i}[\beta(1-r P(1-\gamma))]+y[\alpha+\beta r P(1-\gamma)]}{\alpha+\beta} .
\end{aligned}
$$

Comparing coefficients and solving for $\gamma$ yields the equilibrium of the subgame,

$$
\gamma^{*}=\frac{\beta(1-r P)}{\alpha+\beta(1-r P)} .
$$

In equilibrium, agents with public information choose

$$
a_{i}=x_{i} \frac{\beta(1-r P)}{\alpha+\beta(1-r P)}+y \frac{\alpha}{\alpha+\beta(1-r P)} .
$$

This implies

$$
\bar{a}=P[\gamma \theta+(1-\gamma) y]+(1-P) \theta
$$

which gives, after some rearrangements

$$
\bar{a}=\theta \frac{\alpha(1-P)+\beta(1-r P)}{\alpha+\beta(1-r P)}+y \frac{P \alpha}{\alpha+\beta(1-r P)} .
$$

This equation shows that, in equilibrium, actions are distorted away from $\theta$ towards $y$. The distortion increases in the precision of public information, $\alpha$, and in the proportion of agents receiving it, $P$ :

- when $\alpha \rightarrow 0, P \rightarrow 0$ or $\beta \rightarrow \infty$, then $\bar{a}=\theta$ : when public information is extremely imprecise or given to almost nobody, or when private information is extremely precise, then public information loses its coordination role and is ignored.

- when $\alpha \rightarrow \infty$ or $\beta \rightarrow 0$, then $\bar{a}=\theta(1-P)+y P$ : when public information is extremely precise or private information extremely imprecise, those who receive public information will disregard private information and choose $a_{i}=y$. The others can only use private signals, which are distributed around $\theta$. Hence, those without public information will choose an average action of $\theta$.

The model of M-S represents a special case of our framework in which $P=1$ is exogenously fixed. Under such circumstances, it is certain that all agents receive a private and a public signal ( $y$ is thus common knowledge among all agents). Here, the unique equilibrium is given by 


$$
a_{i}=\frac{\alpha y+\beta(1-r) x_{i}}{\alpha+\beta(1-r)}
$$

The weight on public information clearly exceeds its weight in $E\left(\theta \mid x_{i}, y\right)$, which is only $\alpha /(\alpha+\beta)$. This mirrors the disproportionate impact of the public signal in coordinating agents' actions. Since there is no other instrument, the only way to restrict the potential damaging effects of public information is a limitation of their precision. Our more general framework provides the central bank with a second instrument that may be superior in reducing the damages of public information.

\section{4 - Welfare implications}

Let us now turn to the first stage of the game and derive the optimal degree of publicity. Since this is our main innovation, we will first calculate the optimal degree of publicity $P$ for precision $\alpha$ being given exogenously, before we focus on the more general solution, in which we solve for the optimal combination of both variables.

\subsection{Optimal degree of information disclosure}

How is welfare affected by the degree of public information disclosure? And what is the interplay between the precision of information and the level of disclosure in terms of welfare effects? Expected welfare is given by

$$
\begin{aligned}
E(W(a, \theta)) & =-E\left[\int_{i \in(0,1)}\left(a_{i}-\theta\right)^{2} d i\right] \\
& =-\int_{i=0}^{P} E\left[\left(\gamma x_{i}+(1-\gamma) y-\theta\right)^{2}\right] d i-\int_{P}^{1} E\left[\left(x_{i}-\theta\right)^{2}\right] d i \\
& =-P\left[\gamma^{2} E\left(\varepsilon_{i}^{2}\right)+(1-\gamma)^{2} E\left(\eta^{2}\right)\right]-(1-P) E\left(\varepsilon_{i}^{2}\right) \\
& =-P \frac{\beta(1-r P)^{2}+\alpha}{(\alpha+\beta(1-r P))^{2}}-(1-P) \frac{1}{\beta}
\end{aligned}
$$

Maximizing expected welfare with respect to $0 \leq P \leq 1$ gives $P^{*}=\min \left\{1, \frac{\alpha+\beta}{3 r \beta}\right\}$. 
The optimal degree of publicity $P^{*}$ is smaller than one if, and only if $\alpha / \beta<3 r-1$. This shows that it is better to disclose public information with a low precision only to a limited audience if coordination is a sufficiently strong motive. For $r<1 / 3$, we always get the corner solution $P^{*}=1$.

Conclusion 1: For all $r \geq 1 / 3$ (sufficiently strong strategic complementarity), the optimal degree of publicity $P^{*}(\alpha, \beta)$ is smaller than 1 , if the private signal is sufficiently precise compared to the public signal.

The intuition for such a result is that a partial disclosure of information can avoid overreaction to a signal, which is potentially far from the true state (when the public signal is imprecise). An imperfect degree of common information disclosure generates a mechanism in which the negative influence of agents' over-reaction is outweighed by the positive impact of coordination on $\theta$.

For a better interpretation, we calculate the relative precision between the two types of signals, for which public information $y$ should be disclosed with probability 1 . The optimal degree of publicity is $P^{*}=1$ if $\alpha+\beta \geq 3 r \beta$. Since $r \leq 1, \alpha \geq 2 \beta$ implies $P^{*}=1$. When the public signal is at least twice as precise as the private signal, public information should be disclosed to all agents with probability 1 . On the other hand, if the private signal $x_{i}$ is extremely precise (so that $\beta \rightarrow \infty$ ), or when the public signal $y$ is extremely imprecise (so that $\alpha \rightarrow 0$ ), then it is optimal to disclose the public signal with a probability of $P^{*} \rightarrow \min \{1,1 /(3 r)\}$.

In the limit, when $\alpha$ approaches zero, public information becomes worthless and will be disregarded even by those who receive it. Thus, for $\alpha=0$, the degree of publicity is irrelevant. However, as soon as public signals have some content ( $\alpha>0)$, the optimal degree of publicity exceeds $1 /(3 r)$ and increases with rising precision $\alpha$.

\subsection{Optimal precision of information}

The impact of the precision of public information on expected welfare is

$$
\frac{\partial E(W)}{\partial \alpha}=P \frac{\alpha+\beta(1-r P)(1-2 r P)}{(\alpha+\beta(1-r P))^{3}} .
$$

Apparently, 


$$
\frac{\partial E(W)}{\partial \alpha} \geq 0 \Leftrightarrow \frac{\alpha}{\beta} \geq(1-r P)(2 r P-1)
$$

The sign of $\partial E W / \partial \alpha$ is ambiguous. If $2 r P>1$ and private information is sufficiently precise, an increase in the precision of public information is detrimental to welfare. The case $P=1$ resembles M-S result according to which the precision of public information increases welfare if, and only if $\alpha / \beta \geq(1-r)(2 r-1)$.

If $2 r P<1$ or if private information is imprecise (small $\beta$ ), then the precision of public information increases welfare.

Conclusion 2: Increasing the precision of the public signal has positive welfare effects if the degree of publicity is sufficiently small.

We also have:

$$
\frac{\partial E(W)}{\partial \beta}=\frac{P(1-r P)\left(\alpha(1+r P)+\beta(1-r P)^{2}\right)}{(\alpha+\beta(1-r P))^{3}}+(1-P) \frac{1}{\beta^{2}} \geq 0
$$

This means that increasing the precision of private information is always a better policy.

Conclusion 3: Increasing the precision of the private signal is always welfare increasing.

If public information can be provided with infinite precision $(\alpha \rightarrow \infty)$, then $y=\theta$ almost certainly and full publication $(P=1)$ leads agents to choose $a_{i}=y$. Thereby, the expected welfare loss is zero, which is the first best solution.

\subsection{Second best optimum for a limited precision of public information}

Inevitable forecast errors limit the precision of public information, which is, after all, just the inverse variance between the public announcement and the ex-post realization of the fundamental state. M-S show that for $P=1$, noisy public information can lead to a higher welfare loss than no public information at all: agents may prefer following a public signal, even of poor quality, because this enhances coordination. However due to the signal's noise, the coordination point is likely to be distorted away from the efficient level $\theta$. The public signal imposes an external effect: it directs all individuals who receive it towards the same action and, thereby, leads to a likely deviation of activities from $\theta$. Such amplification of the initial noise is painful for all agents and damaging for welfare of the society as a whole. 
If the principal has the option to choose the optimal degree of publicity $P^{*}$, then the optimal precision is always maximal. To see this, compare $P^{*}$ with the condition for welfare increasing effects of public information precision. An increase in $\alpha$ raises expected welfare if $\alpha \geq \beta(1-r P)(2 r P-1)$. The optimal degree of publicity $P^{*}$ is less than or equal to $(\alpha+\beta) /(3 r \beta)$, which is equivalent to $\alpha \geq \beta\left(3 r P^{*}-1\right)$. Since $\left(3 r P^{*}-1\right)>\left(1-r P^{*}\right)\left(2 r P^{*}-1\right)$, we conclude that an increase in $\alpha$ always raises expected welfare if the degree of public information is chosen optimally.

Whenever the principal faces upper limits to the possible precision of public information, such that $\alpha \in[0, \bar{\alpha}]$, then the second best solution is to provide public information with the highest possible precision $\bar{\alpha}$ and release it to a proportion $P^{*}(\bar{\alpha})$ of all agents. The optimal degree of publicity is smaller than 1 if $\bar{\alpha}$ is sufficiently small. Whenever $\bar{\alpha}$ is so small that M-S's conclusion applies for $P=1$, then $P^{*}(\bar{\alpha})<1$. But, even if $\bar{\alpha}$ is larger, as argued by Svensson (2005), the optimal degree of publicity may be less than one.

As a consequence, we can state the main result of the paper: even if the precision of public information is restricted by some $\bar{\alpha}$, the central bank should provide public information with maximal (feasible) precision, but with some probability $P$ that is below 1 if $\bar{\alpha}$ is sufficiently small.

Main theorem: The second best optimal policy for $\alpha \in[0, \bar{\alpha}]$ is given by $\alpha^{*}=\bar{\alpha}$ and

$$
P^{*}=\min \left\{1, \frac{\bar{\alpha}+\beta}{3 r \beta}\right\} \text {. }
$$

We summarize these findings in Figure 1 (for $r>1 / 2$ ). Solid curves represent social welfare contours in the $(\alpha, P)$-space. Arrows indicate the direction of increasing welfare. The lower broken line is $P^{*}(\bar{\alpha})$. The upper broken curve indicates the set of points above which an increase in $\alpha$ reduces welfare. Whenever $\bar{\alpha}<\beta(3 r-1)$, the optimal degree of publicity is smaller than one. 
Figure 1 - Social welfare contours in $(\alpha, P)$-space

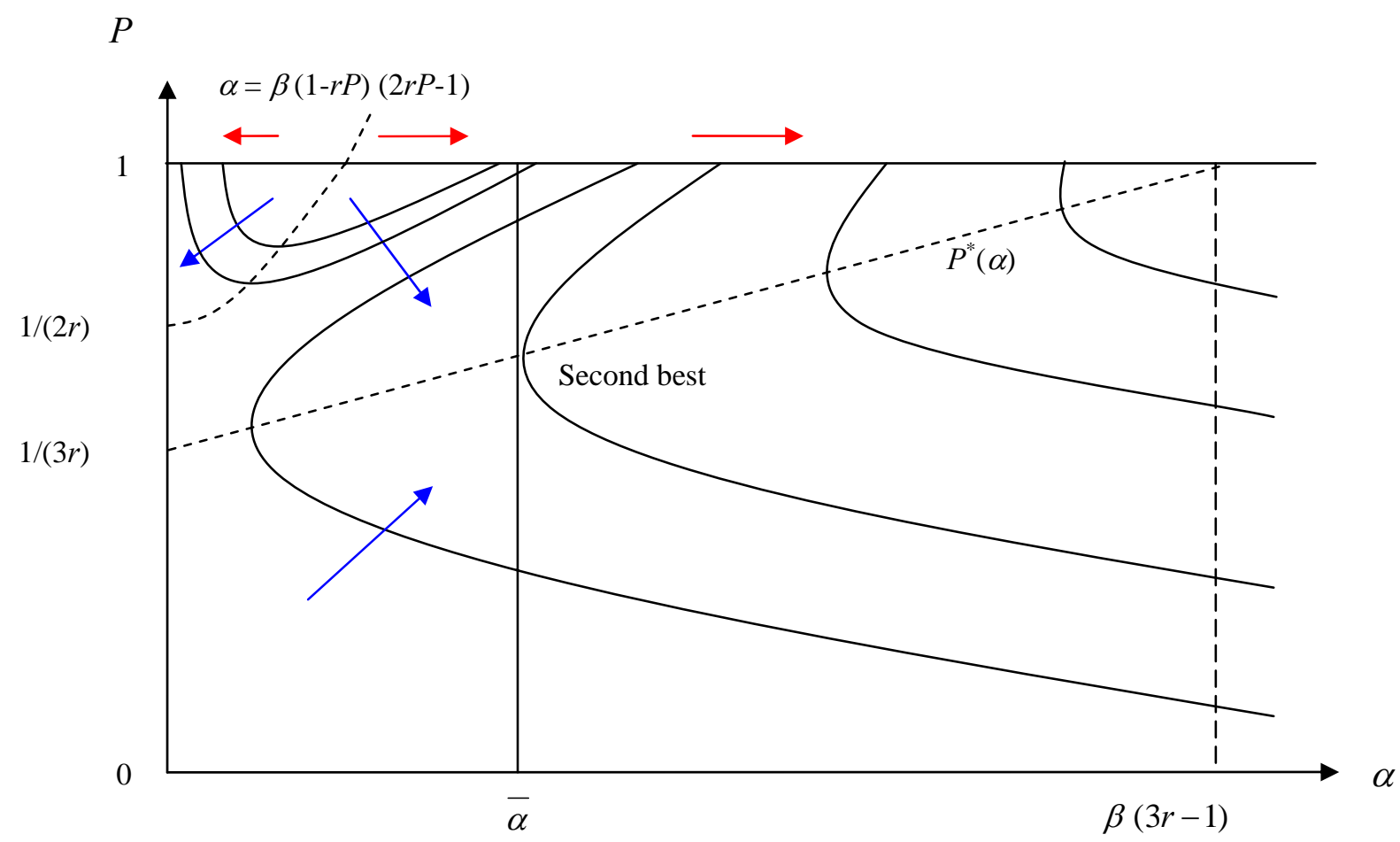

If the central bank cannot avoid that its announcements become common knowledge among all agents $(P=1)$ and cannot raise the public signal's precision above some upper boundary, no information ( $\alpha=0$ ) may be better than maximum precision ( $\bar{\alpha}$ ). M-S (p. 1529) conclude that "even if the choice of $\alpha$ entails no cost, we will see a "bang-bang" solution to the choice of optimal $\alpha$ in which the social optimum entails either providing no public information at all (...) or providing the maximum feasible amount of public information".

Such a ("bang-bang") result does not hold anymore, once we relax the assumption that public signals are received by all agents with certainty. The tool of limiting the degree of publication allows for the exploitation of the positive feature of precise as possible public information: those who receive the public signal can improve their decisions, while withholding information entirely waives these profits for all agents. 


\section{5 - Policy Recommendations and Discussion}

We have shown that a central bank should rather limit the degree of publicity than the precision of information. To what extent can the central bank control the degree of publicity and by which means can it provide partially public information? Public information is a statement that is common knowledge. To achieve common knowledge, one might assemble all agents in one room and announce a statement or publish it in a medium that is common knowledge to be recognized by all agents. In practice, it seems more difficult to achieve common knowledge among all agents than amongst a smaller group. It may not be feasible to assemble all economic actors at the same time and, although all professional traders acknowledge the leading financial newspapers, they read these papers at different times. Hence, they can never be sure that everybody else has the same information.

On the other hand, in coordination games it may be in the agents' own interest to spread their information in order to move others' actions towards their own. Hence, any announcement that is originally released to a limited audience may find its way to a wider public. An agent's action may also be observable by others and reveal private or partially public information. Furthermore, if markets are efficient, any information that is shared by a positive mass of agents has a significant impact on the market and may be revealed by observable prices. Such propagation of information raises the degree of publicity above the original proportion of informed traders, and optimal information policy must account for these multiplier effects.

In this section, we first suggest by which means a central bank can release information to a limited audience. Second, we discuss incentives and mechanisms by which private and partially public information may spread. Finally, we analyse whether a central bank might improve expected welfare by providing information to agents in private instead of publishing a common signal.

\subsection{Means for achieving limited publicity}

There are several means by which central banks release information. Most important are a central bank's own publications (hardcopies and Internet), press releases, press conferences, speeches and interviews. The general practice is that publications and press releases are distributed as widely as possible to get through to all market participants. In addition, publication time is announced beforehand, so as to make sure that everybody has the chance for receiving new information at the same time. Speeches and interviews, instead, are directed 
first of all to those who are physically present plus eventual listeners, if a speech is broadcasted. To reach a wider audience and avoid misinterpretation, the texts of important speeches are also disclosed and sometimes released via the Internet. For interviews, central banks invite journalists of leading financial newspapers to allow for the widest possible impact. None of these channels guarantees common knowledge, but there are clear attempts to achieve the highest possible degree of publicity.

To strive for a more limited degree of publicity, central banks may

(i) provide information to media that are known to be read by a limited audience,

(ii) invite a limited number of journalists for providing new information,

(iii) abstain from broadcasting or publishing speeches,

(iv) communicate with a small number of banks,

(v) hide information in the Internet,

(vi) disclose information at irregular times,

(vii) release information that can be interpreted only by a fraction of speculators.

Launching information in selected media may be a very efficient way of controlling the degree of publicity. Circulation of newspapers is well documented and may serve as a measure for the number of recipients. At least at the IMF, it is common practice to release information to a small number of journalists from selected media. Speeches may also be a very effective tool for achieving partial publicity, because attention to speeches is limited in comparison to written information. Walsh (2005, pp. 3-4) argues that: "Partial announcements can occur when, for example, central bankers make speeches about the economy that may not be as widely reported as formal announcements would be. Speeches and other means of providing partial information play an important role in the practice of central bankers, and these means of communication long predated publication of inflation reports. Speeches, like academic conferences, can be viewed as one means of providing information to only a limited subset of the public." As any other important information, a speech is covered by media afterwards, which increases publicity. These multiplier effects must be considered. However, as will be argued in more detail below, private dissemination of information is less likely generating common knowledge than a direct publication by the central bank.

Central banks seem to disclose some information strategically. For example, central banks often communicate with a small number of banks who thus receive more information than others. For the purpose of limiting publicity, it may help not to announce such meetings or the 
identity of receivers of information to the public either. The timing of announcements is also chosen deliberately. Imagine that the Bank of England makes an announcement during daytime in Europe. Traders may trade in Europe. But, by the time some other stock markets open, prices may have integrated the information and it may be too late for speculators from other areas to trade. Announcements tend to be public in the geographic area where they are released and are, thus, directed at a subgroup of traders in global markets.

Information that is provided via the Internet is, in principle, publicly available. Depending on the transparency of a website, information can be attained more or less easily. The more difficult it is to find certain data, the less likely will they be found by any particular agent. Even if some trader finds information of general interest that is hidden in the depth of a central bank's website, his subjective probability for another person finding the same information will be lower than for information that is reported on the entry page. This lower probability is all that is required for avoiding damaging effects of higher-order beliefs.

Releasing information at irregular times may not prevent that everybody receives the information eventually. But, if publication time is not common knowledge, the information cannot become common knowledge at any point in time either. The same argument applies to private dissemination of information. Although multiplier effects cannot be avoided, secondary distribution of information is less predictable and is likely to have lower effects on higher-order beliefs than primary information. As long as there is no common knowledge about the process, by which private agents spread information, the information cannot become common knowledge either.

Under certain conditions, it may be possible to release signals that can only be interpreted in combination with ex-ante information under the disposal of a limited fraction of market participants. In these cases a public release of the signal amounts to limited publicity. Ambiguous statements do, in general, not yield this effect. With different ex-ante information, an ambiguous statement amounts to an imprecise signal for traders with low ex-ante information. Our results show, however, that imprecise signals should not be released to a general audience. Hence, ambiguity works only, if there is a clear distinction between agents, for whom the ambiguity is resolved, once they combine the statement with their ex-ante information, and agents who have no use at all for the ambiguous signal.

Far from being perfect, all these means of communication should allow the central bank to provide partially public information when the quality of this information is for some reason poor (e.g. forecast errors). Another issue is how the observation by traders of prices and 
others' action might impede the central bank's goal to achieve limited publicity. In a rational expectations equilibrium, markets may integrate private and partially public information such that this information is revealed by the price system. In a fully revealing equilibrium, all information is common knowledge. We think of such an equilibrium as the outcome of a process during which traders move prices. The actions are carried out before the equilibrium is established and a revealing price system can be observed. During this process, it is an advantage to predict the average opinion better than others. Once information is integrated and the price system is settled, there are no gains from speculation anymore. Full revelation requires that all actions have been carried out. Keynes' beauty contest example is meant to represent the behaviour of professional traders who try to make money from speculation during price adjustments. They cannot use information that is revealed only after prices adjust to their actions.

Experimental evidence on herding games, like Kübler and Weizsäcker (2003), indicate that subjects do not fully deduce information from observable actions. In their experiment, subjects had to decide between two actions and could buy some noisy signal containing information about which choice is likely to be the better one. In equilibrium, the first subject would buy this information and reveal it by her decision, while the others would follow the first player's choice, because they can deduce her information from her decision. Actual behaviour, instead, is consistent with a model in which most subjects rationally integrate original signals and take into account that most others respond rationally to original signals. However, they hardly account for higher-order beliefs arising from the consideration that other players do also account for others' rationality. The experiment demonstrates that observing private agents' actions is no substitute for official information. In addition, Heinemann, Nagel and Ockenfels (2004) and Cornand (2005) present experiments on coordination games with private and public information. Contrary to theoretical predictions, they find only small differences in behaviour in response to the mode of information. Other publications in experimental economics ${ }^{3}$ also support the view that common information does not necessarily induce common knowledge.

The practical means, by which the effects on higher-order beliefs can be separated from the provision of information, are not fully understood yet. There may be even better ways to release information, but to a limited audience, if higher-order beliefs could be managed separately. The best mode of information provision would be a full release of the public signal

\footnotetext{
${ }^{3}$ See, for example, Smith (1991) and Nagel (1995).
} 
to everybody, as long as each player believes that he is the only one, who gets the signal. However, this seems impossible and partial publicity is a compromise - a second-best solution given the restraints on mechanisms for providing information.

\subsection{Private provision of public information and information diffusion}

Agents may be able to disseminate a partially public signal to a wider audience by sharing information with other agents. This might make it difficult for the central bank to limit the degree of publicity. Lack of credibility or of common knowledge about the dissemination process may prevent common knowledge of privately provided public signals. There are good reasons to believe that privately provided information is less likely to become common knowledge than official information provided by the central bank.

However, agents may not even be interested in spreading the partially public signal. Raising the degree of publicity has positive and negative effects on an agent's expected payoff. By raising $P$, more agents receive signal $y$ and all agents receiving this signal increase the weight $1-\gamma$ that they put on this signal. This improves coordination with other informed agents. But, other informed agents can improve their coordination as well. An agent's utility depends on the difference between his own coordination failure and the average coordination failure of others. This reflects the zero-sum game in Keynes' beauty contest: an investor gains from predicting the average opinion better than other agents. An agent who spreads a partially public signal $y$ weakens his competitive advantage. In addition, for higher $P$, the agent will put a larger weight on $y$, so that his action is further away from his expected realization of the fundamental state $\theta$. This hurts the goal of meeting fundamentals. Together, the larger distortion of one's action from expected fundamentals and the competition effect may outweigh the coordination advantage associated with spreading one's information.

The net effect of $P$ on the expected payoff (ex post, conditional on receiving the public signal) is ambiguous. Simulations ${ }^{4}$ indicate that conditional expected payoff is decreasing in $P$ for all $P \geq P^{*}$ if the partially public signal is more precise than private information $(\alpha>\beta)$ or if $r<2 / 3$. We found that an increase in $P$ raises an informed agent's expected utility only if the precision of private signals is higher than the precision of the public signal, the coordination motive is sufficiently strong and, in addition, the agent's private signal is far away from the public signal, that is, $\left|x_{i}-y\right|$ is large compared to standard deviations of

\footnotetext{
${ }^{4}$ Simulations are available at http://www.sfm.vwl.uni-muenchen.de/heinemann/publics/optimal-publicity.html.
} 
signals. Only then, an agent may have an incentive to increase the degree of publicity of signal $y$ beyond $P^{*}$.

But, no agent would want to fully disseminate the signal to the whole population. In Appendix B, we show that a marginal increase in the degree of publicity unambiguously reduces an informed agent's expected utility if $P$ is close to 1 . That is,

$$
\left.\frac{d E\left(u_{i} \mid x_{i}, y\right)}{d P}\right|_{P \rightarrow 1}<0 \quad \text { for all } r, \beta \text { with } P^{*}(r, \beta)<1 \text { and for all } x_{i}, y \text {. }
$$

This result indicates that no agent who receives the public signal is interested in full publicity by spreading the signal to everybody. Simulations do also indicate that the posterior expected utility for an informed agent, given $P^{*}$, is always higher than the posterior utility for $P=1$. Thus, if informed agents can only choose between full publicity and not spreading information at all, they would abstain from disseminating the partially public signal.

If there are agents who expect profiting from an increase in $P$, their private and public signals differ considerably. These agents have conflicting interests. Meeting the fundamental requires an action close to $E\left(\theta \mid x_{i}, y\right)$. The beauty contest calls for deviating towards $y$. The trade-off is affected by $P$, because a higher degree of publicity increases gains from deviating towards $y$. When such an agent spreads the public signal, he attributes a larger weight to this signal in his own action, due to the coordination motive. Thereby, his action deviates even further from the expected fundamental. The additional losses from meeting the fundamental must be over-compensated by expected gains from competing in the beauty contest. The agents' incentive to spreading information comes from expected gains that arise when previously uninformed agents choose actions that are closer to his own. If he could choose, which information to provide, he would not spread the true public signal, but rather pretend that the public signal is identical with his action, thus, a linear combination of the true signal $y$ and his private signal $x_{i}$. That is, he has an incentive to misrepresent the public signal instead of truthfully sharing it with others. This should be expected by those who receive public signals from private sources. However, these receivers do not know, in which direction the signal is distorted, because $x_{i}$ is private information. Accounting for the incentives to misrepresent a public signal, amounts to a lower precision of the privately provided public signal. Privately provided signals are not as credible as information provided by the central bank. Lack of credibility, however, prevents that other agents put the same weight on such signals as on official information. Thus, there is a crucial difference between official signals 
and those provided by private agents: the latter may always be biased and, therefore, appear less credible. This limits the capacity of private agents to spread partially public information and raise the degree of publicity too far beyond the original $P^{*}$.

If we follow Svensson (2005), realism requires assuming that the partially public signal is more precise than private information $(\alpha>\beta$ ). In this case, agents who can be sure to receive the partially public signal are interested in a degree of publicity that is lower than socially optimal. If they could alter the degree of publicity, they would rather reduce than increase it. Therefore, we may expect that insiders, like private banks with close ties to the central bank, might try to convince the central bank to limit public disclosures at a level below $P^{*}$.

\subsection{Central bank's provision of private information}

Instead of releasing a common signal, the central bank might want to release information to agents in private. Within a speculative-attack game, Heinemann and Illing (2002) suggest that the central bank should release information to each agent privately with some idiosyncratic noise, thereby avoiding common knowledge. In their speculative-attack game, private information has the advantage of preventing multiple equilibria that may be interpreted as potential currency crises out of self-fulfilling beliefs.

Does such a mechanism improve expected welfare in our beauty-contest game and would it be optimal to release these idiosyncratic signals to all agents in the economy? To answer this question, suppose that the central bank, instead of releasing her own information $y$, gives a fraction $P$ of all agents an official signal $y_{i}=y+v_{i}$, where random terms $v_{i}$ are i.i.d. with variance $\sigma_{v}^{2}$. For $\sigma_{v}^{2} \rightarrow 0$, official signals are almost as precise as the common signal $y$ in the reference model. They help informed agents to predict fundamentals, which is welfare enhancing. However, official signals are very close to each other and, thereby, serve as focal points for coordinating activities almost as much as in the reference model with a common official signal. Idiosyncratic official signals are distributed around the central bank's information. If this information is noisy (small $\alpha$ ), official signals may distort informed agent's actions away from their expected fundamentals, which was the cause for the detrimental welfare effect in the reference model. Idiosyncratic official signals have about the same effect as a common signal, if the correlation of official signals is common knowledge. The equilibrium weight on these signals is somewhat smaller than on a common signal (due to the additional noise) and it rises in $P$ as in the reference case. Thereby, allowing the possibility of providing idiosyncratic official signals instead of a common signal does not 
alter the main result that these signals should be provided to some fraction of the population close to $P^{*}$. And they should be as precise as possible, bringing us back to a common signal.

The discontinuity of the equilibrium manifold, known from speculative-attack games, does not apply to the beauty-contest framework with its unique equilibrium. Not common knowledge of the signal per se is responsible for the suboptimal high weight that agents attribute to it. The detrimental welfare effects also arise if official signals are private but distributed around imprecise central bank information, provided that the correlation of private signals is common knowledge.

\section{6 - Conclusion}

Our paper contributes to an ongoing debate about the desirability of increased transparency. In financial markets, speculators gain from predicting the average opinion better than others. Thus, agents not only have an interest in responding to the underlying fundamentals but also have a coordination motive to take similar actions. Their actions represent strategic complements. Nevertheless, at the social level, effective market coordination per se is not socially valuable. Efficiency requires that the price system reflects market fundamentals and the central bank aims at driving agents' actions as close as possible to these fundamentals. In this context, the strong focal potential exerted by public information can be welfare damaging because it induces overreactions, if public signals lack precision.

This paper distinguishes two dimensions of information disclosure policy: precision of signals and degree of publicity. Both instruments interact. We have shown that in a context where the central bank makes inevitable forecast errors, it is efficient to provide public information with highest possible precision to only a fraction of market participants. The optimal degree of publicity is rising in the precision of signals. If public signals can be released with a precision that is at least twice the precision of private information, public signals should be released to all traders.

The intuition behind these results is the following: A public signal serves as focal point for traders in predicting other's actions. They attach a larger weight to these signals than justified by their informational content. Thereby, traders' actions are heavily influenced by public information. If this information is imprecise, agents coordinate with a large probability on a state that differs substantially from fundamentals. This reduces ex-ante expected welfare. However, all information is valuable to the extent that it helps predicting the state of the 
world. A limited degree of publicity reduces incentives to exaggerate the weight on public signals. Thereby, limiting publicity combines the positive effects of valuable information for those who get it with a confinement of its threats by limiting the number of receivers. The higher the precision of public signals, the lower is the probability that an exaggerated weight reduces welfare. Hence, the optimal degree of publicity is rising to one if public signals are sufficiently more precise than private information.

Publishing information in selected media, speeches, conferences, interviews, and meetings with representatives of selected financial institutions are effective means of providing partially public information. These means play an important role in the actual policy of central banks already. Central banks seem to disclose information strategically in communicating with a small number of banks and to deliberately choose the timing of announcements. Thereby, our results give a rationale for the common practice of central banks to release partially public information in addition to official publications. Our main result shows that these means of partial publicity should only be employed for announcements of low precision.

While agents may be able to disseminate a partially public signal to a wider audience, we have shown that this is, in general, not in their interest, because spreading their information reduces their competitive advantage in predicting average opinion better than others. If an agent can expect to profit from sharing his information with others, he has an incentive to misrepresent the partially public signal. Thus, privately provided public signals are less credible than official information and exert a smaller effect on coordinating actions.

Nevertheless, there are some obvious implementation problems to our policy advice. Even though it is already in central banks' practice to partially disclose information, the issue of the determination of the actual degree of publicity - including estimates of multiplier effects that are generated by media coverage and private dissemination of signals - needs to be addressed. Laboratory experiments indicate that common signals do not generate all of the higher-order beliefs that are so detrimental to our model economy. It is an open question whether and how mechanisms of providing information can separately affect real traders' beliefs about the state of the world and higher-order beliefs about others' expectations. A rigorous experimental analysis of this question might be useful for designing optimal mechanisms of disseminating information to financial markets. 


\section{Appendix A}

In Section 3 we claimed that an equilibrium strategy is a linear combination of the available signals. Otherwise, there might be multiple equilibria. Linearity can be proven along the lines suggested by Morris and Shin (2002). The optimal action of agent $i$ is given by

$$
a_{i}=(1-r) E_{i}(\theta)+r E_{i}(\bar{a}) \text {. }
$$

For agents who receive just a private signal, $a^{i}=x^{i}$. For the others, the optimal strategy depends on both signals and is denoted by $a_{p}^{i}\left(x^{i}, y\right) \in\left[x^{i}, y\right]$. The average activity is given by

$$
\bar{a}=P \int_{0}^{1} a_{p}^{i}\left(x^{i}, y\right) d i+(1-P) \int_{0}^{1} x^{i} d i=P \bar{a}_{p}(\theta, y)+(1-P) \theta,
$$

where $\bar{a}_{p}(\theta, y)$ is the average action of informed agents. Hence,

$$
\begin{aligned}
a_{p}^{i}\left(x^{i}, y\right) & =(1-r P) E_{i}(\theta)+r P E_{i}\left(\bar{a}_{p}(\theta, y)\right) \\
& =(1-r P) E_{i}(\theta)+r P(1-r P) E_{i}\left(\bar{E}_{p}(\theta)\right)+(r P)^{2} E_{i}\left(\bar{E}_{p}\left(\bar{a}_{p}(\theta, y)\right)\right), \quad j \neq i \\
& =(1-r P) \sum_{k=o}^{\infty}(r P)^{k} E_{i}\left(\bar{E}_{p}^{k}(\theta)\right)+\lim _{k \rightarrow \infty}(r P)^{k} E_{i}\left(\bar{E}_{p}^{k}\left(\bar{a}_{p}(\theta, y)\right)\right) .
\end{aligned}
$$

$\bar{E}_{p}^{k}(\theta)$ denotes the average expectation of the average expectation $\ldots$ (k-times) of $\theta$, and $\bar{E}_{p}^{k}(\bar{a})$ is the average expectation of the average expectation ... of the average action, where all averages concern informed agents only. For $r P<1$, the limes term is zero. Morris and Shin (2002) have shown that $E_{i}\left(\bar{E}_{p}^{k}(\theta)\right)$ is a linear combination of $x^{i}$ and $y$. Hence, $a_{p}^{i}\left(x^{i}, y\right)$ is also a linear combination of $x^{i}$ and $y$.

QED

\section{Appendix B}

Here, we prove our claim from Section 5.2 that for $P \rightarrow 1$,

$$
\frac{d E\left(u_{i} \mid x_{i}, y\right)}{d P}<0
$$


Recall that for an informed agent $a_{i}=\gamma x_{i}+(1-\gamma) y$, while $a_{j}=x_{j}$ for an agent who does not receive the partially public signal. Furthermore, $x_{i}=\theta+\varepsilon_{i}$ and $y=\theta+\eta$. The payoff for agent $i$ is

$$
u_{i}=-(1-r)\left(a_{i}-\theta\right)^{2}-r \int_{0}^{1}\left(a_{j}-a_{i}\right)^{2} d j+r \int_{0}^{1} \int_{0}^{1}\left(a_{j}-a_{k}\right)^{2} d j d k
$$

Using the equations above, $a_{i}-\theta=\gamma \varepsilon_{i}+(1-\gamma) \eta$. Without loss of generality, we assume that agents $j \in[0, P]$ receive the public signal and $j \in(P, 1]$ are not informed. Then,

$$
a_{j}-a_{k}=\left\{\begin{array}{ccc}
\gamma\left(\varepsilon_{j}-\varepsilon_{k}\right) & \text { if } & j, k \leq P \\
\gamma \varepsilon_{j}+(1-\gamma) \eta-\varepsilon_{k} & \text { if } & j \leq P \wedge k>P \\
\varepsilon_{j}-\varepsilon_{k} & \text { if } & j, k>P .
\end{array}\right.
$$

Inserting these terms, the payoff of an informed agent is

$$
\begin{aligned}
u_{i}=-(1-r)[\gamma & \left.\varepsilon_{i}+(1-\gamma) \eta\right]^{2}-r P\left[\gamma\left(\varepsilon_{i}-\varepsilon_{j}\right)\right]^{2}-r(1-P)\left[\gamma \varepsilon_{i}+(1-\gamma) \eta-\varepsilon_{j}\right]^{2} \\
& +r P^{2}\left[\gamma\left(\varepsilon_{j}-\varepsilon_{k}\right)\right]^{2}+2 r P(1-P)\left[\gamma \varepsilon_{j}+(1-\gamma) \eta-\varepsilon_{k}\right]^{2}+r(1-P)^{2}\left[\varepsilon_{j}-\varepsilon_{k}\right]^{2} .
\end{aligned}
$$

Up to now, we did not normalize units of account. To ease calculations, we use this degree of freedom here and normalize $\alpha+\beta=1$. Expectations of agent $i$ conditional on his information are denoted by $E_{i}$. Using the rules for conditional expectations of normally distributed variables and the above normalization, we get

$$
\begin{array}{ccc}
E_{i}\left(\varepsilon_{i}^{2}\right) & =1+\alpha^{2}\left(x_{i}-y\right)^{2}, \\
E_{i}\left(\eta^{2}\right) & =1+\beta^{2}\left(x_{i}-y\right)^{2}, \\
E_{i}\left(\varepsilon_{i} \eta\right) & =1-\alpha \beta\left(x_{i}-y\right)^{2}, & \\
E_{i}\left(\varepsilon_{j}^{2}\right) & =1 / \beta & \text { for } j \neq i, \\
E_{i}\left(\varepsilon_{i} \varepsilon_{j}\right)=E_{i}\left(\varepsilon_{j} \varepsilon_{k}\right)=E_{i}\left(\varepsilon_{j} \eta\right)=0 & \text { for } j, k \neq i .
\end{array}
$$

Agent $i$ 's conditional expected payoff is now given by

$$
\begin{aligned}
E_{i}\left(u_{i}\right)= & -(1-r)\left[\gamma^{2} E_{i}\left(\varepsilon_{i}^{2}\right)+2 \gamma(1-\gamma) E_{i}\left(\varepsilon_{i} \eta\right)+(1-\gamma)^{2} E_{i}\left(\eta^{2}\right)\right]-r P \gamma^{2}\left[E_{i}\left(\varepsilon_{i}^{2}\right)+1 / \beta\right] \\
& -r(1-P)\left[\gamma^{2} E_{i}\left(\varepsilon_{i}^{2}\right)+2 \gamma(1-\gamma) E_{i}\left(\varepsilon_{i} \eta\right)+(1-\gamma)^{2} E_{i}\left(\eta^{2}\right)+1 / \beta\right] \\
& +2 r P^{2} \gamma^{2} / \beta+2 r P(1-P)\left[\gamma^{2} / \beta+(1-\gamma)^{2} E_{i}\left(\eta^{2}\right)+1 / \beta\right]+2 r(1-P)^{2} / \beta
\end{aligned}
$$




$$
\begin{aligned}
= & -1+\frac{r}{\beta}+r P\left[(1-\gamma)^{2}(1-1 / \beta)+2(1-P)(1-\gamma)^{2}\right] \\
& -\left[(1-r P)(\gamma-\beta)^{2}+r P\left(\gamma^{2}(1-\beta)^{2}-2(1-P)(1-\gamma)^{2} \beta^{2}\right)\right]\left(x_{i}-y\right)^{2} \\
= & A+B\left(x_{i}-y\right)^{2} .
\end{aligned}
$$

Taking the derivative of the expected payoff with respect to $P$ and rearranging terms yields

$$
\begin{aligned}
& \frac{d E_{i}\left(u_{i}\right)}{d P}=A^{\prime}+B^{\prime}\left(x_{i}-y\right)^{2}, \text { where } \\
& A^{\prime}=-r\left[\left(1-\gamma^{2}\right)(1 / \beta-1)+2(1-\gamma)^{2}(2 P-1)\right]+2 r P[\gamma(1 / \beta-1)-2(1-P)(1-\gamma)] \gamma^{\prime}, \\
& B^{\prime}=r\left[(\gamma-\beta)^{2}-\gamma^{2}(1-\beta)^{2}-2(2 P-1)(1-\gamma)^{2} \beta^{2}\right] \\
& -[2(\gamma-\beta)+2 r P \beta(1-2 \gamma+2 \beta(1-P)+\beta \gamma(2 P-1))] \gamma^{\prime},
\end{aligned}
$$

and $\gamma^{\prime}$ denotes the derivative of the weight on the private signal to $P$. With a continuum of agents, there will be agents with any value for $x_{i}-y$ almost certainly. To ensure that no agent has an incentive to raise the degree of publicity, $A^{\prime}$ and $B^{\prime}$ must be negative. Using $\gamma=\frac{\beta(1-r P)}{1-\beta r P}$ and $\gamma^{\prime}=\frac{-(1-\beta) \beta r}{[1-\beta r P]^{2}}$, we can check the signs of these terms by calculating them for a fine grid of parameter values. It turns out that $A^{\prime}<0$ for all $\beta, r, P \in(0,1)$. Function $B^{\prime}$ is increasing in $\beta$ and $r$ and decreasing in $P$. If $\beta<1 / 2$ or $r<2 / 3, B^{\prime}$ is negative for all $P \geq P^{*}$. For all $\beta$ and $r, B^{\prime}$ becomes negative if $P \rightarrow 1$.

QED

\section{References}

Angeletos G.-M. and A. Pavan (2004), “Transparency of Information and Coordination in Economies with Investment Complementarities,” American Economic Review (Papers and Proceedings), 94, 2, 91-98.

Amato J. D., S. Morris and H. S. Shin (2002), "Communication and Monetary Policy," Oxford Review of Economic Policy, 18 (4), 495-503, Winter.

Amato J. D. and H. S. Shin (2006), "Imperfect Common Knowledge and the Information Value of Prices,” Economic Theory, symposium issue on monetary policy, 27, 213-241. 
Bannier C. and F. Heinemann (2005), “Optimal Transparency and Risk Taking to Avoid Currency and Liquidity Crises,” Journal of Institutional and Theoretical Economics, 161, 374-391.

Cornand C. (2005), “Speculative Attacks and Informational Structure: An Experimental Study," Review of International Economics, forthcoming.

Cukierman A. (2001), “Accountability, Credibility, Transparency, and Stabilization Policy in the Eurosystem," In Charles Wyplosz (ed), The Impact of EMU on Europe and the Developing Countries (pp.40-75), Oxford University Press.

Faust J. and L. E. O. Svensson (2001), “Transparency and Credibility: Monetary Policy with Unobservable Goals,” International Economic Review, 42, 369-397.

Geraats P. (2002), “Central Bank Transparency,” The Economic Journal, 112, F532-F565.

Heinemann F. and G. Illing (2002), "Speculative Attacks: Unique Sunspot Equilibrium and Transparency,” Journal of International Economics, 58, 429-450.

Heinemann F., R. Nagel. and P. Ockenfels (2004), “The Theory of Global Games on Test: Experimental Analysis of Coordination Games with Public and Private Information,” Econometrica, 72, 1583-1599.

Hellwig C. (2002a), "Public Information, Private Information and the Multiplicity of Equilibria in Coordination Games,” Journal of Economic Theory, 107, 191-222.

Hellwig C. (2002b), "Public Announcements, Adjustment Delays and the Business Cycle,” UCLA, mimeo.

Hellwig C. (2005), “Heterogeneous Information and the Benefits of Transparency,” UCLA, mimeo.

Kübler D. and G. von Weizsäcker (2003) “Limited Depth of Reasoning and Failure of Cascade Formation in the Laboratory,” Review of Economic Studies, 71, 425-441

Metz C. (2002), “Private and Public Information in Self-Fulfilling Currency Crises,” Journal of Economics, 76, 65-85.

Mishkin F. (2004), “Can Central Bank Transparency go too far?,” NBER Working Paper 10829, October.

Morris S. and H. S. Shin (1998), “Unique Equilibrium in a Model of Self-Fulfilling Currency Attacks,” American Economic Review, 88, 587-597. 
Morris S. and H. S. Shin (1999), “A Theory on the Onset of Currency Attacks,” in: The Asian Financial Crisis: Causes, Contagion and Consequences, ed. by P.-R. Agenor, M. Miller, D. Vines, and A. Weber. Cambridge, U.K., Cambridge University Press, 230255.

Morris S. and H. S. Shin (2002), “Social Value of Public Information,” American Economic Review, 92, 1522-1534.

Morris S. and H. S. Shin (2005), "Central Bank Transparency and the Signal Value of Prices,” paper prepared for the Fall 2005 meeting of the Brookings Panel on Economic Activity.

Morris S., H. S. Shin and H. Tong (2005), "Response to "Social Value of Information: Morris and Shin (2002) is Actually Pro Transparency, Not Con"," American Economic Review, forthcoming.

Nagel R. (1995), “Unraveling in Guessing Games: An Experimental Study,” American Economic Review, 85, 1313-1326.

Reis R. (2004), “Inattentiveness,” PhD dissertation, Harvard University.

Romer C. D. and D. H. Romer (2000), "Federal Reserve Information and the Behavior of Interest Rates,” American Economic Review, 90, 429-457.

Sims C. (2003), "Implications of rational inattention,” Journal of Monetary Economics, 50, $665-690$

Smith V. L. (1991), “Experimental Economics: Behavioral Lessons for Microeconomic Theory and Policy,” in: Smith, V.L., Papers in Experimental Economics, Cambridge University Press, 802-812.

Svensson L. E. O. (2005), "Social Value of Public Information: Morris and Shin (2002) Is Actually Pro Transparency, Not Con,” American Economic Review, forthcoming.

Walsh C. E. (2005), “Transparency, Flexibility, and Inflation Targeting,” University of California, First draft, September.

Winkler B. (2000), "Which Kind of Transparency? On the Need for Clarity in Monetary Policy Making,” E.C.B. Working Paper Series, 26, August.

Woodford M. (2005), “Central-Bank Communication and Policy Effectiveness,” NBER working paper 11898. 